\title{
ARAMIS: Toward a Hybrid Approach for Human- Environment Interaction
}

\author{
Stefano Carrino ${ }^{1}$, Elena Mugellini ${ }^{1}$, Omar Abou Khaled ${ }^{1}$, and Rolf Ingold ${ }^{2}$ \\ ${ }^{1}$ College of Engineering and Architecture of Fribourg, Switzerland \\ \{Stefano.Carrino, Elena.Mugellini, Omar.AbouKhaled\}@Hefr.ch \\ ${ }^{2}$ University of Fribourg, Switzerland \\ Rolf.Ingold@Unifr.ch
}

\begin{abstract}
In this paper we describe ARAMIS a novel hybrid approach aiming to enhance the human smart-environment interaction. We define this approach as hybrid since it is the combination of three different dichotomies: wearable and pervasive computing paradigms, virtual and real worlds, optical and nonoptical sensing technologies. In order to validate the proposed approach we have designed a multimodal framework, in which gestures have been chosen as the main interaction modality. The framework design aims firstly to efficiently manage and merge information from heterogeneous, distributed sensors and secondly to offer a simple tool to connect together such devices. Finally a prototype has been developed in order to test and evaluate the proposed approach.
\end{abstract}

Keywords: Hybrid gesture recognition, pervasive computing, human-computer interaction, multimodality.

\section{Introduction}

The interaction between the human and the environment changes along with the technological evolution of society and, with the rise of post-desktop human computerinteraction models such as the pervasive and wearable paradigms, our daily behavior is being changed further. Nowadays specialized research branches are analyzing the interaction between people (Human Centered Interaction) and technological environments [1]. On the one hand lots of works aim at improving the quality of life of inhabitants of smart environments, dealing with the design of new kinds of interactions [2] [3] and trying to enrich the normal way of interacting with the physical environment by technologies seamlessly embedded in everyday objects of our lives [4]. On the other hand the wearable computing paradigm improves the human-computer interaction in a complementary way focusing on: privacy, personalization, and freedom of interaction [5]. In fact, wearable devices can be more intimately and actively involved in the user's activity, and potentially become intelligent personal assistants [6]. Finally with the enhancement of mobile devices performances (processing power, bandwidth and autonomy), it becomes possible to develop new approaches for realtime hybrid systems. In such kind of systems mobile and fixed devices can share information and combine elaboration capabilities and technologies [3]. 
The major contribution presented in this paper is the proposition of a novel hybrid approach, called ARAMIS, to enhance the human interaction with the surrounding environment. Our method is defined hybrid since it is based on three different dichotomies: environmental/wearable paradigms, real/virtual worlds, optical/non-optical sensing technologies. Coupling these technologies ARAMIS aims to exploit the strong points of individual technologies and overcome their weakness (such as light condition in vision-based techniques).

Finally since the gestures are a natural way for a person to interact with the environment and the objects around him [2] [7], we decided, in the context of this research project to consider them as the main human-environment interaction modality.

The paper is organized as follows: Section 2 describes the state of the art of related researches. Section 3 describes and discusses the proposed hybrid approach. Section 4 presents the framework architecture. Section 5 introduces the prototype end the system evaluation. Finally, section 6 concludes the paper and discusses future research plans.

\section{Related Projects}

Multidisciplinary researches are trying to define what the environment can sense [1] and how it has to react to implicit interactions (with special focus on context awareness) [2] [8] [9]. At the same time, approaches based on on-body, wearable technologies have been widely studied since they can be easily designed to address several typical problems of ubiquitous computing such as: to provide user identification, to supply personal feedback, to improve user activity or user command recognition, etc. [10] [11]. However autonomy, intrusiveness, processing power, ad hoc and not standardized communication protocols still are the major drawbacks of these approaches; even if at present day several works are trying to address these different issues [12].

Both pervasive and wearable computing paradigms have advantages and disadvantages. However several features can be combined in a complementary fashion in order to improve the system performance [5] and the quality of the interaction. According to our knowledge the jointly use of these paradigms, coupled with mixed reality environments and large spectrum of sensing technologies is not largely studied. Moreover the realization of a framework managing this heterogeneous set of sensing data could help researcher in the HCI field to prototype their applications.

\section{ARAMIS Hybrid Approach}

In order to clarify our model some definitions are needed. In this section we will describe the hybrid approach we proposed, providing definitions and presenting our conceptual structure, stressing the theoretical advantages of this approach.

\subsection{Definitions}

We have called our approach hybrid for three different reasons:

- A coupling of pervasive and wearable paradigms. In this paper we intend as pervasive paradigm all the information processing (acquisition, transfer, elaboration and presentation) managed at environment level, whereas as wearable the information processing on the human. 
- A coupling of real and virtual worlds (object and tools). Real elements could be bound to enhanced digital counterpart. Interactions with virtual objects can change the state of real ones and vice versa. This augmentation of the environment around the user makes possible new kinds of interactions. For instance a user can virtually extend his arms and interact with remote augmented tools.

- A coupling optical and non-optical sensing technologies. Cameras, gyroscopes, accelerometers, and other sensors useful for gesture interaction and context awareness are employed in order to increase the robustness of the dataset available for processing.

\subsection{Discussion}

Merging together several approaches can allow the system to take advantages of different approaches and technologies. Pervasive and wearable paradigms allow bringing the technology in the environment and on the human itself. A mixed reality environment increases the interaction possibilities beyond the limits of material world and physics rules. Finally combining more acquisition technologies make possible to have a more robust and accurate dataset. So far, several technologies (inertial, acoustic and optical) have been investigated. Those studies showed that each technology has its own advantages and drawbacks and usually it is adapted to specific use cases. For instances optical technologies give very useful information but are expensive in term of processing power and, in addition, sensible to lighting changes; whereas inertial sensors are more resistant to environmental changes but suffer for systematic and incremental errors. Based on these observations, we propose a framework seamlessly integrating several technologies that can be used in a complementary or alternative fashion. For instance we can use both the inertial and optical technology in a redundant manner to provide information about the user gestures or we can use them in a complementary fashion; the elaboration of the images acquired from a camera (expensive in terms of processing requirements) will be processed at environmental side whereas the inertial sensors will be analyzed by the machine on the user.

A strong point that needs to be further stressed in the proposed hybrid approach is how to merge the advantages of the pervasive and the wearable paradigms. The table 1 (an extension of the researches presented in [5]) compares the two approaches resuming their main features.

Table 1. Features provided by Pervasive and Wearable paradigms

\begin{tabular}{c|cc}
\hline FEATURES & PERVASIVE & WEARABLE \\
\hline Privacy & & $\mathbf{X}$ \\
Personalization & & $\mathbf{X}$ \\
Consistency & & $\mathbf{X}$ \\
\hline Localized information & $\mathbf{X}$ & \\
Localized control & $\mathbf{X}$ & \\
Resources & $\mathrm{X}$ & \\
\hline
\end{tabular}


Privacy: the target, the medium and the result of an interaction are visible only for the interaction performer.

Personalization: interaction that can be personalized for a user.

With the term consistency the prolonged, continuous interaction between the human and the device is taken into account. This exchange of information allows classification algorithms to evolve along with the interaction with the user. In fact an improvement of performances is possible in a twofold manner: the system toward the user and, at the same time, the user toward the system.

Localized information and localized control: information and controls are localized in special spot or interactive areas.

A pervasive approach has, in most of the cases, less restriction in terms of resources (energy, processing power, etc.).

Some of that features, such as personalization and consistency, can be integrated effortless using the pervasive and the wearable paradigm at the same time. However privacy and personalization in the interaction are further increased by our approach. In fact, information can be fitted and displayed for specified users (according to their profiles and interaction context) exploiting virtual elements.

Another strong point in our hybrid approach is the resource management. In fact, information is elaborated in the mobile system or/and in the fixed one, according to the type of information to handle, the available devices and the quality of the connection. For instance, data that is troublesome to transfer, such as a high frequency data from the accelerometers, can be elaborated locally on the wearable machine and just the processed information (e.g. extracted features) is sent further. On the other hand information that is heavy to elaborate (e.g. images) can be elaborated at environmental side.

The ARAMIS framework, presented in the next section, realized to exploit the advantages of coupling these paradigms and technologies aims to be a useful tool for every researcher that need work in multi-paradigm and multimodal domains.

\section{ARAMIS Framework}

In order to validate the described approach there is the necessity of a system able to acquire, transfer, elaborate and present the information coming from heterogeneous, distributed (on the users and the environment) sensors and actuators under real time constraints. To address these challenges and to provide a technical support for our theoretical approach we design a framework able to handle these kinds of issues. Designing the framework we chose to manage data as in multimodal systems. This allows exploiting previous knowledge and results from multimodal research area in order to design, prototype and evaluate our approach with widely accepted methods.

Moreover this framework can be a valid aid for researcher working with sensors LAN or WAN in different contexts providing a flexible structure to connect sensors and classification algorithms. In next paragraphs our framework is described taking into account the gesture as main interaction modality, whereas extensions toward other modalities will be briefly discuss. 
ARAMIS framework components are presented in figure 1. The Environment Data Module (EDM) and On-Body Data Module (ODM) are the interfaces with the user. They are basically components working as drivers. These modules acquire data from sensors and elaborate them for further transmissions and elaborations. EDM and ODM handle contextual information related to the environment and the user, describing the interactive devices available in the environment, what services they offer and how to interface with them.

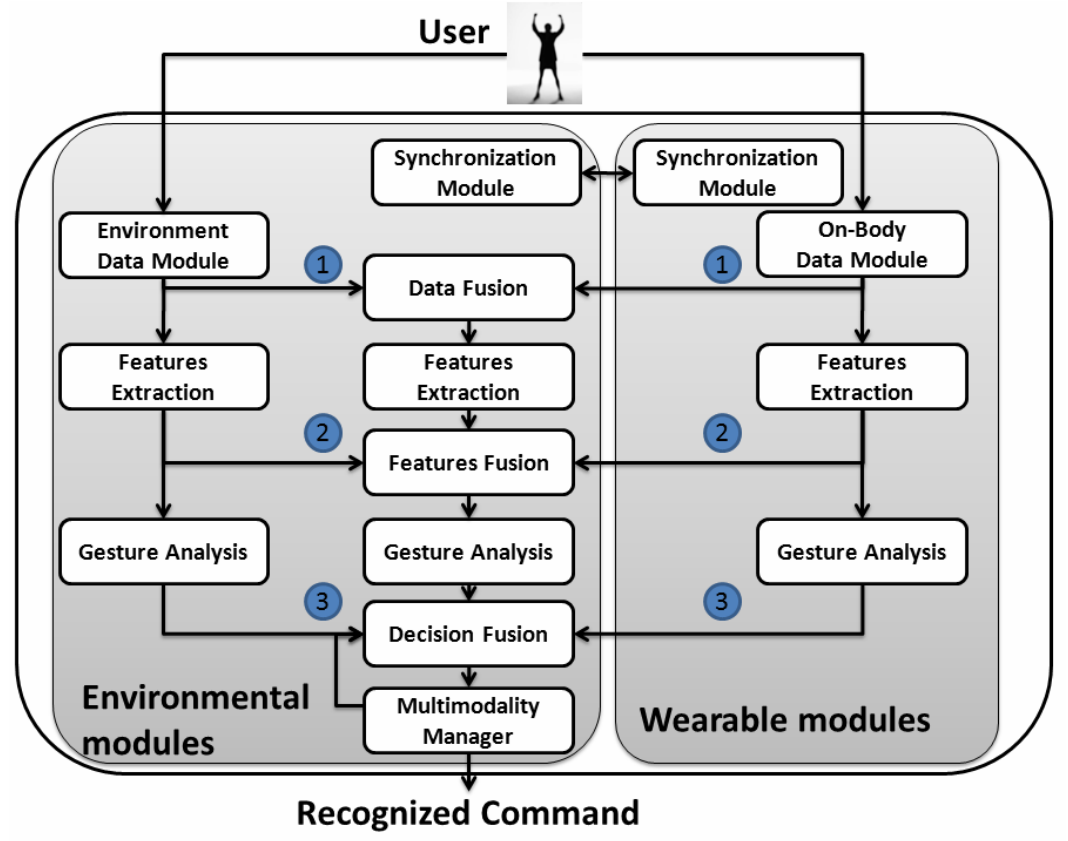

Fig. 1. ARAMIS framework - Overview

According to our hybrid approach the EDM and the ODM represent, respectively, pervasive and wearable components.

As shown in fig. 1, the information originated from each sensor can follow three data flows (numbered in the figure). In the first case, raw data are immediately merged in the Data Fusion module (data level fusion [13]). In the second one, information from the environment and the wearable devices is elaborated separately: features are extracted (Features Extraction modules) and then merged (feature level fusion). In the last case, separated Gestures Analysis modules perform the data classification; hence gestures are recognized separately and finally merged in the Decision Fusion module (decision level fusion).

In addition Data Fusion, Features Fusion and Decision Fusion modules have a double goal: firstly to merge the information and secondly to synchronize it. In fact the information is produced from different sources, typically working asynchronously and at different data rates. 
The framework allows organizing the information according one of the three configurations for each sensor. Although this allows great flexibility to a system developed using our framework, the drawback is in the realization of the fusion and decision modules. Changes in the system configuration will comport subsequent modifications in these modules or in the use of more complex and often less valid algorithms. At the moment, this selection is done statically by evaluating the available sensors, the target application and the gesture recognition performances needed.

The fig. 1 highlights the case of gesture recognition; nonetheless any modality can be acquired and processed according to the same schema. Hence, even if it is not shown in the figure, the Multimodality Manager module will handle the fusion of the different modalities at decision level.

\subsection{Wearable and Pervasive Paradigms as Modalities}

From a conceptual perspective, the framework handles sensing data as in a standard multimodal system. This choice grants the possibility to bring the knowledge developed in the multimodal HCI field directly into systems implemented using this framework. For example models as CARE and CASE [13] previously linked to the multimodal research domain can be used to design and characterize systems developed with the ARAMIS framework. The CASE model specifies the multimodal communication types at machine-side. Therefore information from the two paradigms can be used in the system in a concurrent way (e.g. two distinct tasks in parallel, no co-reference), in alternate way (e.g. a task with temporal alternation of modalities), in synergistic way (e.g. a task, in parallel, using several co-referent modalities) or exclusive way (one task after the other using one modality at time, no co-reference) (CASE model). The CARE model specifies system usability properties as complementarity (multiple modalities are to be used within a temporal window to reach a given state), assignment (only one modality can be used to reach a given state), redundancy (multiple modalities, here the pervasive and wearable paradigm, have an equivalent expressive power and are used in the same temporal window) and equivalence (it is necessary and sufficient to use any one of the available modalities). These models have been used to prototype and in the future to evaluate our approach.

\section{ARAMIS Prototype and Evaluation}

A first version of the framework has been developed in the context of a smart living room. This environment provides rich and interactive features where many devices are able to communicate and interact among each other [14].

Due to the high number of tasks involved in the realization of the whole system (multimodality, gesture recognition, system synchronization, etc.) in the first development phase we focus on demonstrating the feasibility of our approach. Afterward an evaluation of the prototype has been performed in order to assess two main aspects: flexibility of the system, measuring the improvement in the elaboration time using the ARAMIS framework in several configurations, and the changes in recognition rate of gesture commands in different configurations. 


\subsection{Set-Up and Scenario}

As base for the implementation we chose a set-up allowing the realization of a simplified interaction scenario. In this setting we have as pervasive components: an interactive wall (used as augmented interaction feedback), Bluetooth-controlled switches, and a central processing unit performing the data elaboration task. As wearable components the user has: two wireless accelerometers (worn on the arms), a camera (on one wrist) and an Ultra-Mobile PC (at the belt). The final goal is to interact through gestures with a media center device and blinds to adjust the luminosity in the room.

Exploiting data form the on-body module the system is user-location aware: gestures performed in front of the interactive wall's camera are recognized exploiting both the accelerometers and the camera, whereas a reduced gesture vocabulary performed elsewhere is recognized only by the inertial sensors.

Information between the different components is serialized in XML messages in order to include rich metadata (e.g. a time stamp and device ID are added to data gathered in the EDM and the ODM). Hence, a clock synchronization algorithm has been developed to ensure a known global time. The used algorithm (inspired by [15]) guarantees an average error in the clock synchronization below $3 \mathrm{~ms}$ for our system. It means a clock difference of around 10ms running the synchronization algorithm each 20 minutes (to compensate the CPUs clock shift).

\subsection{Environmental Module}

At environmental side our gesture vocabulary is focused on dynamic gesture recognition, realized with bare hands, based on computer vision techniques (in front of a camera and facing the wall, fig. 2). The software manages a video stream following these steps: data acquisition, hand segmentation, hand tracking, gesture segmentation and finally hand gesture recognition.

The whole gesture recognition process is realized following the next main steps. Firstly, hands are segmented by skin color. Colors are extracted either statically using the LAB color space (robust against light changes [16]) or dynamically executing a face detection algorithm (based on a Haar cascade classifier [17]) and recognizing nose and cheeks. Then, the two hands and the face are tracked using CAMshift algorithm [18]. Gestures are segmented exploiting kinetic features of hands and finally gestures are recognized through a direction vectors approach. In this prototype we use

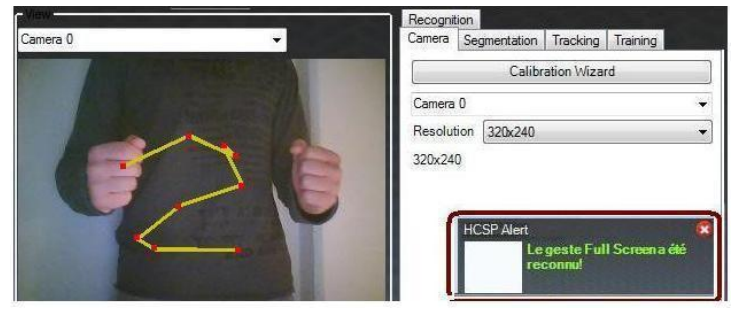

Fig. 2. Environmental module - Hand gesture recognition 
a simple gesture vocabulary of height commands: sweep left, right, up, down, both with one hand and two hands). A visual feedback of the hand tracking process is presented to the user.

\subsection{Wearable Module}

A wearable embedded module for gesture recognition has been developed (fig. 3 shows the sensors on the user arm). Data, received by several sensors (in our set-up inertial sensors and a camera) are sent to an Ultra-Mobile PC. This device works as a bridge with a main server. This module can be configured to work according any of the three paths presented above: as data router (path 1), extracting features from the raw data (path 2), or recognizing gestures and sending the result to the central unit (path 3). Four gestures are recognized (rotations of the wrist, rotations of the wrist two times, shakes of the hand in two directions, pointing gesture). To realize the pointing gesture a customized version PTAMM algorithm (presented by Castel et Al.[19]) has been used. Our implementation allows the tracking in a three dimensional space of the user position related to some interactive objects and tools. Hence this technique has two interesting outcomes: firstly it is possible to make contextaware applications based on the user position (in this scenario the system knows when the user is facing the wall-camera). Secondly it becomes possible to manage the user identification.

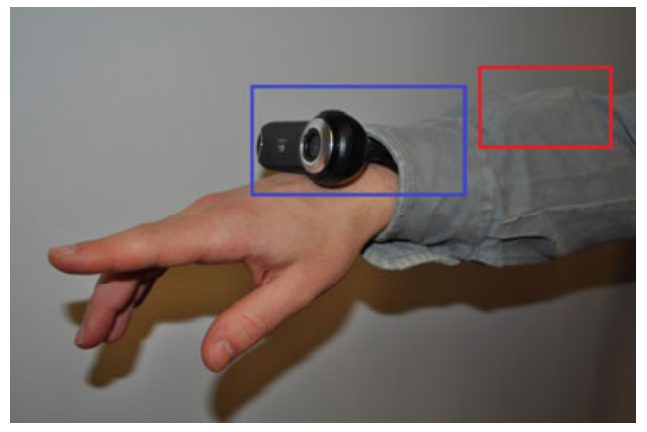

Fig. 3. Wearable sensors. Indigo (left) - camera. Red (right) - inertial sensors.

\subsection{Evaluation and Results}

In the evaluation phase we have concentrated our efforts to demonstrate the feasibility of the proposed approach deferring usability evaluation to a second test phase. From a technical point of views we evaluate the systems paying attention to real-time issues. We tested our system obtaining elaboration time between 150 and $750 \mathrm{~ms}$ according to the configuration adopted. The most of this time is spent by the system sending data (serialization, transfer, and deserialization) between devices on the user and in the environment. In the best configuration $(150 \mathrm{~ms}$ between the gesture performance and the feedback) was still perceived as an acceptable response time by the users in our interaction scenario. However, at this moment, the proposed gesture language needs more assessments to be deeply evaluated independently of the worn devices (still too cumbersome). 
Differently from the time performances, the gesture recognition rates did not show significant variations according the system configuration adopted. In the context of our set-up we consider these results as not surprising but deeply tests with a more complex gesture vocabulary are planned for the future.

\section{Conclusion}

In this paper we proposed a novel hybrid approach to enhance the interaction between the human and smart environments. The proposed approach exploits different paradigms and techniques at the same time: pervasive and wearable computing, a mixed reality world to enhance the interaction, and optical and non-optical technologies. In order to validate our approach we designed a framework and we realize a first prototype based on it. Results of the assessments were encouraging: gesture interaction exploiting our hybrid approach can be performed in real time, taking advantage from wearable and pervasive sensors to realize new kind of interactions.

As next steps we plan to perform quantitative measures about system usability and increasing, at the same time, the easiness to connect new sensors to the framework using a service based approach; from that we will complete the formalization of the presented interaction perspective defining a general multimodal language (vocabulary and semantic) suitable for the described approach in a precise scenario.

\section{References}

1. Krumm, J., Shafer, S.: How a smart environment can use perception. On Sensing and Perception, 1-5 (2001)

2. Cook, D.J., Das, S.K.: How smart are our environments? An updated look at the state of the art. Pervasive and Mobile Computing 3, 53-73 (2007)

3. Satyanarayanan, M.: Pervasive computing: Vision and challenges. IEEE Personal Communications 8, 10-17 (2001)

4. Cook, D., Das, S.: Smart Environments: Technology, Protocols and Applications. Wiley Series on Parallel and Distributed Computing (2004)

5. Rhodes, B., Minar, N.: Wearable computing meets ubiquitous computing: Reaping the best of both worlds. In: International Symposium on Wearable (1999)

6. Pentland, a.: Smart rooms, smart clothes. In: Proceedings of Pattern Recognition, pp. 949953 (2002)

7. Bolt, R.a, Herranz, E.: Two-handed gesture in multi-modal natural dialog. In: Proceedings of the 5th Annual ACM Symposium on User Interface Software and Technology - UIST 1992, pp. 7-14 (1992)

8. Schmidt, A.: Implicit human computer interaction through context. Personal Technologies 4, 191-199 (2000)

9. Schmidt, a.: There is more to context than location. Computers 23, 893-901 (1999)

10. Culler, D.: TinyOS: Operating System Design for Wireless Sensor Networks. Sensors (2006)

11. Nixon, P., Wagealla, W., English, C., Terzis, S.: Security, privacy and trust issues in smart environments. In: Smart Environments: Technologies, Protocols and Applications. WileyInterscience, Hoboken (2005) 
12. Argyroudis, P.G., O'Mahony, D.: Securing communications in the smart home. Embedded and Ubiquitous Computing, 133-139 (2004)

13. Dumas, B., Lalanne, D.: Multimodal interfaces: a survey of principles, models and frameworks. Human Machine Interaction (2009)

14. Mugellini, E., Khaled, O.A., Pierroz, S.: Generic Framework for Transforming Everyday Objects into Interactive Surfaces. Human-Computer (2009)

15. Römer, K.: Time synchronization in ad hoc networks. In: Symposium on Mobile Ad Hoc Networking and Computing (2001)

16. Cai, J.: Detecting human faces in color images. Image and Vision Computing 18, 63-75 (1999)

17. Papageorgiou, C.P., Oren, M.: A general framework for object detection. In: Sixth International Conference on Computer Vision (IEEE Cat. No.98CH36271), pp. 555-562 (1998)

18. Bradski, G.: Computer vision face tracking for use in a perceptual user interface. Interface (1998)

19. Castle, R.O., Klein, G., Murray, D.W.: Combining monoSLAM with object recognition for scene augmentation using a wearable camera. Image and Vision Computing 28, 1548-1556 (2010) 